\title{
Non-epithelial ovarian carcinoma: what is the optimal staging surgery?
}

\author{
Heriberto Medina-Franco, Luis Enrique Colonna-Márquez \\ Department of Surgery, Section of Surgical Oncology, National Institute of Medical Sciences and Nutrition "Salvador Zubirán", Mexico City, \\ Mexico \\ Contributions: (I) Conception and design: H Medina-Franco; (II) Administrative support: H Medina-Franco; (III) Provision of study materials or \\ patients: LE Colonna-Márquez; (IV) Collection and assembly of data: All authors; (V) Data analysis and interpretation: All authors; (VI) Manuscript \\ writing: All authors; (VII) Final approval of manuscript: All authors. \\ Correspondence to: Heriberto Medina-Franco, MD, FACS. Department of Surgery, Section of Surgical Oncology, National Institute of Medical \\ Sciences and Nutrition "Salvador Zubirán”, Vasco de Quiroga 15, Colonia Sección XVI, Tlalpan, Mexico City 14000, Mexico. \\ Email: herimd@hotmail.com.
}

\begin{abstract}
Non-epithelial ovarian neoplasms are a group of infrequent, heterogenous clinical and histological tumors that account from $6 \%$ to $10 \%$ of ovarian malignancies, the two most common non-epithelial ovarian neoplasms are germ cell tumors and sex cord-stromal cell tumors, each of these classifications is divided in multiple histologic subtypes. In the case of epithelial ovarian tumors comprehensive surgical staging has remained as the gold standard for staging, nonetheless for non-epithelial ovarian neoplasms the use of staging surgery has remained debatable and controversial throughout the years in order to correctly stage non-epithelial ovarian carcinomas. Despite the fact that the role of surgery remains critical in the adequate management of all ovarian tumors, there are several manners to surgically approach an ovarian tumor depending on the type of tumor, risk factors and staging of disease. There are multiple reasons why the use of surgery in non-epithelial ovarian tumors is still debatable nowadays, for instance the main reason relies in the low prevalence of this disease, therefore there are few studies that actually offer a clear and sufficient overview to this issue. The objective of this manuscript is to present a comprehensive review of the non-epithelial carcinoma and to focus in the latest information, evidence and recommendations about the optimal treatment and staging surgery for these tumors.
\end{abstract}

Keywords: Ovarian carcinoma; germ cell tumors; sex cord tumor; staging surgery

Submitted Jan 13, 2020. Accepted for publication Jul 06, 2020.

doi: $10.21037 /$ cco-20-18

View this article at: http://dx.doi.org/10.21037/cco-20-18

\section{Introduction}

Non-epithelial ovarian tumors are usually composed of germ cell tumors, sex cord tumors, metastatic tumors, raresarcomas and lipoid cell tumors, in this review the primary focus will be on germ cell and sex cord-stromal tumors. The origin of non-epithelial ovarian tumor arises from specific type of cell within the ovary, like germ cells, granulosa cells, theca cells, steroid cells and stroma fibroblasts, nonetheless non-epithelial tumors may arise less frequently from nonspecific ovarian cells for example mesenchymal cells.
Malignant ovarian germ cell tumors (MOGCTs) derived from primordial germ cells and compromise about $5 \%$ of all ovarian malignancies seen typically in 20-30-year-old women, they are diagnosed at an early stage of disease and usually have unilateral presentation (1). Table 1 presents the WHO 2014 classification of germ cell tumors. Ovarian sex cord-stromal tumors account for approximately $3-5 \%$ of ovarian neoplasms; histologic subtypes are adult or juvenile granulosa cell, Sertoli-Leydig and sex cord tumors with annular tubules or others not specified. Characteristically sex cord tumors manifest with endocrine symptoms and the 
Table 1 Germ cell tumors classification (WHO 2014)

Dysgerminoma
Yolk sac tumor
Embryonal carcinoma non-gestational choriocarcinoma mature
teratoma
Immature teratoma
Mixed germ cell tumor

This classification is of public domain.

Table 2 Sex cord-stromal and steroid cell tumors classification (WHO 2014)

Pure stromal tumors
Fibroma
Cellular fibroma
Thecoma
Luteinized thecoma associated with sclerosing peritonitis
fibrosarcoma
Sclerosing stromal tumor
Signet-ring stromal tumor
Microcystic stromal tumor
Leydig cell tumor
Steroid cell tumor
Steroid cell tumor, malignant
Pure sex cord tumors
Adult granulosa cell tumor (AGCT)
Juvenile granulosa cell tumor
Sertoli cell tumors
Wex cord tumor with annular tubules
Wixed sex cord-stromal tumors
With heth heteroleydig cell tumors
Wifferentiated Moderately differentiated
Wifologous elements Poorly differentiated
Withents Sex cord-stromal tumors, NOS

WHO classification is of public domain.

most common of them the granulosa cell tumor traditionally presents in postmenopausal women and middle age, with an incidence rate between 4 per 1,000,000 and 2 per 1,000,000 women for germ cell tumors and sex cord-stromal tumors, respectively (2). Sex cord-stromal tumors are a low-grade malignancy with few histological exceptions like the poorly differentiated sarcomatoid form. Table 2 shows the WHO 2014 classification of sex cord-stromal and steroid cell tumors (3). A different subtype is the small cell carcinoma of the ovary producing hypercalcemia that composes less than $1 \%$ of ovarian carcinomas and the median age at diagnosis is 24 years (4).

The clinical presentation of non-epithelial ovarian cancers is diverse, commonly manifested with pelvic pain, pelvic or abdominal mass and menstrual irregularities. Diagnosis studies usually include computed tomography (CT) scan of thorax, abdomen and pelvis, pelvic ultrasound and positron emission tomography scan in some germ cell tumors (5). Serum studies that should be obtained in the work up of young patients include alpha-fetoprotein (AFP), cancer antigen 125 (CA-125), lactate dehydrogenase (LDH) and serum human chorionic gonadotropin (HCG), full blood count, renal and liver function test. Specific markers for disease are inhibin B for granulosa cell tumors and nonspecific markers that are useful for prognostic information are preoperative levels of HCG, AFP, LDH and CA-125 (6). Nowadays the use of CA-125 in non-epithelial ovarian tumors is limited to MOGCTs, this carbohydrate antigen that is present in normal epithelium of the female reproductive system and other organs was described first described in 1983 as a biomarker for epithelial ovarian cancer and has remained since then as the most widely used tumor biomarker for the detection of epithelial ovarian cancer, on the other hand since non-epithelial ovarian cancer usually lack the epithelium that expresses CA-125 other markers like HCG, AFP and LDH remain as the standard depending on the histology of tumor. However, there have been reports of non-epithelial tumors that express increased serum CA-125 level like the one from Sekiya et al. that in 1997 reported for the first time five patients with pure yolk sac tumor with elevated CA-125 that correlated with the extent of the disease (7). In 2018 Kim et al. analyzed 161 patients from the medical databases of Asan Medical Center with MOGCTs in which an elevated level of CA-125 (>249.5 U/mL) was significantly associated with worse overall survival (8). Nonetheless this study had a few pitfalls in it's statistical analysis that was addressed later in a letter to the editor, thus it's reliability remains uncertain. As stated before there is few evidence that supports the use of preoperative CA-125 as a prognostic factor in non-epithelial ovarian tumors, specially MOGCTs, 
Table 3 Components of staging in macroscopically early ovarian cancer

Visual inspection
Evaluation of ovarian disease: unilateral or bilateral
Evaluation of the surface of the ovary
Evaluation of the capsule of the ovary
Evaluation of disease spillage
Histopathologic evaluation
All suspicious lesions
Random biopsies of peritoneum
Cul de sac
Right abdominal gutter
Left abdominal gutter
Undersurface of diaphragm
Infracolic omentectomy
Pelvic lymph nodes
Para-aortic lymph nodes
Peritoneal washings or ascites

thus there is a need to make more studies to evaluate the use of CA-125 as a prognostic factor in malignant ovarian cell tumors. Nowadays the only real use of CA-125 in non-epithelial ovarian cancer as the European Society for Medical Oncology (ESMO) recommends is to measure HCG, AFP, LDH, CA-125 and inhibin B only for patients undergoing chemotherapy for surveillance of response evaluation and follow up.

\section{Overview of surgical staging in non-epithelial ovarian cancer}

During the last 40 years there has been a huge progress in the prognosis of ovarian carcinoma patients, as a result of the improvement in surgical techniques and medical advancements. Historically in 1972 Bagley et al. (9) reviewed for first instance the issue of staging of ovarian cancer, reporting a $30 \%$ discrepancy between staging by non-oncologists and reoperation by gynecologic oncologists, furthermore classic studies like the one from McGowan et al. (10) in 1985 that found that in 291 patients with ovarian cancer only $54 \%$ received proper staging and the one from Mayer et al. in 1992 that found a higher survival rate in patients early epithelial ovarian tumors who were comprehensively staged versus with the ones who underwent a more limited surgical procedures (11) reinforcing the use of comprehensive surgical staging as the gold standard of care for early-stage ovarian cancer. The staging system for non-epithelial ovarian cancers is extrapolated from the surgical staging system for epithelial ovarian tumors defined by the International Federation of Gynecology and Obstetrics (FIGO) in 1985. Staging laparotomy is composed of complete surgical staging (CSS) and optimal cytoreduction if it is necessary, followed by platinum-based chemotherapy. Before modern chemotherapy, prognosis of patients with diagnosis of nondysgerminomas germ cell tumors was poor, nonetheless with the introduction of chemotherapeutic regimens like bleomycin/etoposide/cisplatin (BEP) in the 1980s longterm survival is the usual outcome in all patients with early or advanced-stage dysgerminomas and non-dysgerminomas MOGCTs. It's important to address that for certain nonepithelial ovarian tumors like dysgerminoma stage IA and immature teratoma stage I the recommended treatment is surgery alone. Despite the fact that chemotherapy has been tremendously important in improving the prognosis of malignant ovarian germ cell carcinoma, surgery is vital in the staging and treatment of the disease (12). Multiple ways exist to perform a surgical approach either an open route or in selected cases minimally invasive approaches like laparoscopy and robotics may be an option. The first laparoscopic report of partial surgical staging of an early ovarian cancer appeared in 1990 (13) and if feasible nowadays laparoscopic surgical approach must be preferred when compared with laparotomy (14). Finally it's important to address the main elements of comprehensive surgical staging (Table 3) which includes an exhaustive examination of the abdominal cavity, unilateral salpingo-oophorectomy, infracolic omentectomy, biopsy of the diaphragmatic and paracolic gutter peritoneum, inspection of para-aortic and pelvic lymph nodes and peritoneal washings in case of no macroscopic disease dissemination (15).

\section{Surgical staging in malignant ovarian tumors}

Since MOGCTs are extremely rare, the basis of the diagnosis of microscopic disease by comprehensive surgical staging for germ cell tumors in adults was derived from the management of epithelial ovarian cancers. It is clear and undebatable that evidence stablishes comprehensive surgical staging in epithelial ovarian cancer as the standard of care, nonetheless actual evidence is debatable over this 
practice in adults with germ cell tumors. For instance, the major questions rely on which is the best approach in apparent early-stage malignant ovary carcinomas. Lin et al. performed a retrospective review of 50 patients with MOGCTs and they observed that omission of surgical staging was associated with an increased risk of disease recurrence, supporting that this surgery is vital to establish the extent of disease, prognostic and to assess postoperative treatment (16). Another retrospective review from Chan et al. evaluated 760 patients from the Surveillance, Epidemiology, and End Results Program, to evaluate the advances made in the surgical approach of MOGCTs, concluding that fertility-preserving surgery with concomitant surgical staging for germ cell cancers is associated with improved survival outcomes (17). Additionally, other aspects to point out is that the ESMO guidelines currently states that routine contralateral ovarian biopsy is not needed when it is macroscopically normal, considered level of evidence III and grade of recommendation A (12). Bilateral germ cell tumors prevalence ranges from $4.3 \%$ to $6.9 \%$ of all MOGCT's (18) usually dysgerminoma and immature teratoma. In this case bilateral involvement embodies a major concern in young patients that desire to preserve fertility. The staging surgery according to the ESMO guidelines consists in preservation of the uterus and of at least a functional part of an ovary because current evidence has not demonstrated that radical initial surgery conveys a benefit to recurrence rate and survival. Unfortunately, in the mayor part of this cases it is not possible to perform this kind of fertility preserving surgery, due to the massive bilateral ovarian involvement that usually is encountered without the possibility to preserve any intact contralateral ovary (1). Therefore, it remains crucial to make a decision based on the extent of risk recurrence, chemotherapysensitivity and the patient's fertility desires. Conservative treatment most remain the cornerstone in these patients due to the high chemosensitivity of this kind tumors (19). Another important point that the ESMO guidelines point out is the fact that in postmenopausal patients, or patients with advanced-stage disease or patients with bilateral ovarian involvement, the recommendation is to perform abdominal hysterectomy and bilateral salpingooophorectomy with CSS (level of evidence III and grade of recommendation A).

It's relevant to discuss that in pediatric patients the concept of CSS as standard of care is still debatable; this is due to the extremely chemosensitivity of these tumors in pediatric population. Several cohorts in pediatric patients have demonstrated that in patients estimated to have early stage disease that did not undergo complete comprehensive surgical staging there was not an increase recurrence rate (20). Billmire et al. reported a pediatric group of 131 children with MOGCT that underwent surgical staging, in which despite $21 \%$ of the patients did not had peritoneal cytology, 36\% did not underwent omentectomy and $97 \%$ did not underwent lymphadenectomy, all of them had a good prognosis with 6-year survival rates $>90 \%$ for all stages, concluding that survival is not compromised by the type of surgical staging performed. Additionally they recommend as a standard surgical approach the following: (I) cytologic evaluation of ascites or peritoneal lavage, (II) careful examination of peritoneal surfaces and biopsy or excision of any abnormalities, (III) direct visual examination and palpation of retroperitoneal lymph nodes with sampling of abnormal ones, (IV) evaluation of the omentum with removal if any adherent or abnormal areas noted, (V) evaluation of the contralateral ovary with biopsy of any abnormal areas, and (VI) complete resection of the involved ovary with preservation of the fallopian tube if not involved (21). Other authors like Lee et al. consider that the current surgical management proposed in these cases alternative to the CSS, is to perform a superficial visual inspection following complete resection of the tumor with sparing of fallopian tube; visual evaluation of contralateral ovary, omentum, lymph nodes, and peritoneal surfaces; biopsy of any suspicious lesions or lymph nodes; and cytologic evaluation of peritoneal washing or ascites (19). The ESMO current guidelines establish that unilateral salpingo-oophorectomy with preservation of the contralateral ovary and the uterus is the standard surgical treatment for young patients with germ cell tumors (12).

It is common to encounter situations where patients have undergone inadequate surgical staging, in these cases, evidence remains controversial amid the best conduct to approach these situations. Some authors state that the need for re-exploration for restaging purposes is questionable, because noninvasive imaging techniques are capable of detecting disease of less than $5 \mathrm{~cm}$, making it highly curable (22). Thus, biopsy of nonpalpable lymph nodes is probably unnecessary. Evidence from MITO-9 retrospective study indicates that surveillance is the best option instead of surgical restaging in these cases (23), intensive follow up surveillance in this consists in regular pelvic and abdominal CT scans as well as periodic measure of tumor markers. The limited benefit in restaging a patient with an inadequate first surgery relies in the principle that 
the treatment for incomplete and positive staging does not change, being the standard of treatment cisplatinbased chemotherapy, on the other hand, surgical restaging confers an increase in treatment burden and psychological distress (22). In the case that surgical restaging is preferred over surveillance, the best way to do it is with minimally invasive technique procedures, it has been demonstrated that laparoscopic procedures in oncologic patients has advantages over conventional surgery, like decreased in the length of hospitalization and faster recovery that enables to start early postoperative chemotherapy (24). Further studies must be done to establish whether restaging or surveillance is the best approach.

Another important and controversial issue regarding MOGCTs is whether routine pelvic and para-aortic lymphadenectomy should be performed or not at the initial surgery. Kumar et al. demonstrated that early lymphadenectomy is associated with increased potential morbidity due to lymphedema (25). Today there are not a consensus about systematic use of lymphadenectomy, nonetheless MITO-9 multicenter Italian retrospective study demonstrated that avoiding some staging peritoneal procedures may increase recurrence rate, without an impact on overall survival (23). Several studies like the one from Kuru $e t a l$. where they analyzed a total of 151 with primary adult type granulosa cell tumor have suggested that lymph node metastasis in patients adult granulosa cell tumor (AGCT) are uncommon and favor the omission of routine pelvic and paraaortic lymph node dissection (26), another study from Ertas et al. that analyzed retrospectively 42 patients with MOGCT concluded that the use of fertility sparing surgery has the same results in terms of recurrence or survival compared with more aggressive approaches like incomplete or complete lymphadenectomy (20), Mahdi et al. also analyzed 1,083 with ovarian germ cell tumors $54 \%$ underwent surgery without lymphadenectomy and $46 \%$ had lymphadenectomy, in the multivariate analysis the presence of lymph node metastasis had no significant effect on survival $(\mathrm{P}=0.16)$ concluding that neither lymph node metastasis or lymphadenectomy are predictors of survival in patients with ovarian germ cell tumors (27). Nonetheless, the prognosis of lymph node metastasis is highly dependent of the histology and usually predicts poor survival (23), for example in yolk sac histology worsens the prognosis. On the other hand, other studies have compared patients who underwent or not lymphadenectomy, and found that that neither lymph node metastasis or performance of lymphadenectomy have an impact on survival. In a retrospective cohort study of MOGCT, Liu et al. (28) compared two groups of 46 patients, one underwent classical comprehensive surgical staging versus the other group that received the staging previously stated in this article described by Billmire et al. (21) founding that survival or rates between the groups were not significantly different. The central reason why systemic lymphadenectomy must be avoided, is because MOGCTs are characteristically highly chemosensitive, therefore even patients who have tumor recurrence, can actually be successfully treated with chemotherapy, conveying an excellent survival despite the completeness of surgical staging (29). The only exception to this recommendation is in patients with early stage disease that otherwise does not require adjuvant chemotherapy because of favorable prognostic factors; in those cases, ESMO guidelines recommend to perform lymphadenectomy when there is evidence of nodal abnormalities during surgical exploration and/or lymphadenopathy in the initial CT scan. Other authors consider the performance of lymphadenectomy crucial in these patients even if there is no evidence of nodal abnormalities at surgical or imaging examination (30). Further retrospective studies like $\mathrm{Hu}$ et al. demonstrate that fertility-sparing surgery and incomplete surgical staging did not have an impact in the prognosis for MOGCTs, claiming that it might be safe to preserve fertility and reduce the number of surgical procedures in malignant ovary germ cell tumors despite the stage or pathology (31).

\section{Surgical staging in sex cord-stromal tumors}

These tumors are mostly found in an early stage, thus having higher survival rates than other malignant ovarian cancers. Being more commonly encountered in women of reproductive age, the staging surgery is also massively influenced by the patient's child bearing desire. The general principle according to the ESMO guidelines is that in early stage child bearing age and preservation of the uterus and contralateral ovary in young patients conveys no increased risk of recurrence (23). The American National Comprehensive Cancer Network 2017 guidelines for ovarian cancer, states that the staging/treatment surgery for early stages IA/IC with fertility desire is fertility-sparing surgery (preservation of uterus and contralateral ovary, along with surgical staging). Nonetheless in certain types of tumors like juvenile granulosa cell tumors, the fact of performing this staging surgery alone specially in stage IC2 and IC3 of disease remains highly debatable, this is 
mainly because one of the few studies that exist evaluating the prognostic factors of granulosa cell tumor made in 2007 by Lee $e t a l$. that investigated the prognosis factors in 35 patients with ovarian granulosa cell tumors finding out that FIGO stage and tumor rupture were the only factors associated with the outcome of GCT (32), on the other hand a case series by Nasioudis et al. that evaluated the prognostic significance of adjuvant chemotherapy for patients with IC granulosa cell tumors in 492 patients using the National Cancer Data Base finding out that approximately 33\% of the patients with stage IC received adjuvant chemotherapy although there was not an associated with a survival benefit (33), thus some authors like the ones from the NCCN guidelines recommend only observation oppositely to the ESMO guidelines that recommend adjuvant chemotherapy in this stage. Apart from this fact, some guidelines also recommend that patients with granulosa cell tumors must undergo additional conservative (endometrial curettage) or radical (hysterectomy) treatment to rule out synchronic uterine cancer, this recommendation is based on several case reports $(34,35)$ that present synchronous adult ovarian granulosa cell tumor and endometrial cancer or hyperplasia, physiologically both tumors growth rely in their hormone-secreting capacity of specially of estradiol, thus having a possible hormone relation between these two neoplasia due to the rarity of granulosa cell tumor more studies need to be carried out to stablish the precise causality between these two tumors. ESMO guidelines do not advocate retroperitoneal evaluation for sex cordstromal tumors in early stages due to the very low incidence of retroperitoneal metastases in this situation, the only indication for lymphadenectomy could be the presence of suspicious or palpable lymph nodes (19).

In 2009 Brown et al. demonstrated in a retrospective chart review in 262 patients that lymph node metastasis in ovarian sex cord-stromal tumors is rare, suggesting that lymphadenectomy may be omitted when staging patients with ovarian sex cord-stromal tumors (36). More recently, in 2017 Nasioudis et al. performed a retrospective populationbased study to establish the prevalence of metastasis to the lymph nodes in 1,156 patients with early stage malignant sex cord-stromal tumors and the effect of performing regional node sampling on their survival: the 5 -year cancer specific survival was very similar in both groups, being $92.7 \%$ for patients who did received lymphadenectomy and $94.7 \%$ for patients who did not received it, concluding that regional lymphatic mode metastasis is uncommon in patients with apparent early sex cord-stromal tumors and that lymphadenectomy does not represents a benefit in survival for this patients (37). As opposed to the previous studies, an analysis of SEER database of 576 patients, most of them with early-stage disease $(69.1 \%$ and $13.7 \%$ stages I and II, respectively) performed by Wang et al. in 2018, investigated the prognostic factors in patients with malignant sex cord-stromal tumors with lymph node dissection and the influence of the proportion between positive and total retrieved lymph nodes (LODDS) on their prognosis; the authors concluded that there are several independent prognostic factors for survival in patients with lymphadenectomy and the ratio between total/retrieved lymph nodes is of value to establish prognosis. Thus, the authors recommend that in patients younger than 50 years, tumor less than 10 centimeters, early tumor stage, granulosa cell type and LODDS $<0.5$ are predictors of improved survival, making it an option to perform a conservative approach for patients with fertility desire and with presence of the previously mentioned factors (38).

\section{Surgical staging in small cell carcinomas of the ovary hypercalcemic type}

These ovarian tumors were described by Dr. Robert Scully about 30 years ago, being an extremely rare pathology in medical literature (39). They are characterized for being a highly aggressive cancer in young women, having an increased risk of extra-ovarian spread hence a very poor prognosis, lymph node metastasis are present in the initial diagnosis in half of the cases (40). In this type of tumor, conventional surgical staging and treatment for all stages relies in surgical approach including hysterectomy with bilateral salpingo-oophorectomy as well as peritoneal and nodal staging surgery. In 1994 Young et al. reported the largest series to date composed by 150 patients with small cell carcinoma and observed reduced survival in patients treated conservatively, of 14 patients who had surgery that included bilateral salpingo-oophorectomy $57 \%$ survived without recurrence, in contrast to only $23 \%$ of the patients who had a unilateral salpingo-oophorectomy $(\mathrm{P}=0.075)$ concluding that there was a better outcome after a more extensive operation for stage IA tumors (4). On 2009 Dykgraaf $e t$ al. reported a patient with this variant of ovarian tumor FIGO stage IIIC treated with conservative surgery followed by chemotherapy, interval debulking surgery and local radiotherapy that had a follow-up of 60 months without evidence of disease, thus proposing conservative surgery in an advance stage of disease (41). 
ESMO guidelines base their recommendations in the results of the series of Young et al. and recommend radical surgery in all stages (level of evidence IV, grade of recommendation A). The actual recommended treatment relies in adjuvant treat after radical surgery with high dose chemotherapy and radiotherapy, making the potential preservation of gonadal function unachievable even if one ovary and uterus are preserved (12).

It is crucial to emphasize that no full-term pregnancy has been ever reported in current literature after this type of conservative surgical management (4). This is the main reason why guidelines recommend radical surgery and lymphadenectomy even though there is a lack of evidence evaluating staging and therapy strategies. The current hope in treatment of this tumor relies on data suggesting a tumor suppressor role of SMARCA4 making it a possible key therapeutic vulnerability in SMARCA4-deficient cells in the future (42).

\section{Conclusions}

It is clear that there is still need to perform multiple prospective randomized controlled trials to establish sufficient evidence that fertility sparing surgery and omission of CSS is the gold standard for clinical management MOGCT, meanwhile current evidence, as previously stated, support recommendations towards fertility sparing surgery and omission of complete staging patients with MOGCT. Similar for sex cord-stromal tumors fertility sparing surgery remains feasible for young women desiring child-bearing preservation in early stages IA and IB, controversial still for stage IC. Guidelines and the few literature that exists regarding mall cell carcinomas of the ovary hypercalcemic type, recognize it as rare-aggressive unique type of nonepithelial ovarian cancer, in which radical surgery must be performed in all patients, despite the stage or fertility desire of the patient. Without a doubt, it's important to emphasize that due to the low prevalence of these neoplasms current evidence that supports the fertility sparing staging surgery and omission of lymphadenectomy is scarce. In the case of MOGCTs several authors established the Malignant Germ Cell International Collaborative group and are currently working in analyzing a large international database in order to establish a consensus on manage for germ cell tumors in pediatrics and adult patients (43). Hence, there is a great need to perform follow-up, intensive and multicenter studies to establish and analyze the precise risks and benefits of these procedures and strengthen the actual recommendations provided by the diverse international guidelines.

\section{Acknowledgments}

Funding: None.

\section{Footnote}

Provenance and Peer Review: This article was commissioned by the Guest Editor (Heriberto Medina-Franco) for the series "Ovarian Cancer" published in Chinese Clinical Oncology. The article was sent for external peer review organized by the Guest Editor and the editorial office.

Conflicts of Interest: Both authors have completed the ICMJE uniform disclosure form (available at http://dx.doi. org/10.21037/cco-20-18). The series "Ovarian Cancer" was commissioned by the editorial office without any funding or sponsorship. HMF served as the unpaid Guest Editor of the series, and serves as an unpaid editorial board member of Chinese Clinical Oncology from Jan 2019 to Dec 2020. The other author has no other conflicts of interest to declare.

Ethical Statement: The authors are accountable for all aspects of the work in ensuring that questions related to the accuracy or integrity of any part of the work are appropriately investigated and resolved.

Open Access Statement: This is an Open Access article distributed in accordance with the Creative Commons Attribution-NonCommercial-NoDerivs 4.0 International License (CC BY-NC-ND 4.0), which permits the noncommercial replication and distribution of the article with the strict proviso that no changes or edits are made and the original work is properly cited (including links to both the formal publication through the relevant DOI and the license). See: https://creativecommons.org/licenses/by-nc-nd/4.0/.

\section{References}

1. Brown J, Friedlander M, Backes FJ, et al. Gynecologic Cancer Intergroup (GCIG) consensus review for ovarian germ cell tumors. Int J Gynecol Cancer 2014;24:S48-54.

2. Schultz KAP, Harris AK, Schneider DT, et al. Ovarian sex cord-stromal tumors. J Oncol Pract 2016;12:940-6.

3. Parkinson CA, Hatcher HM, Ajithkumar TV. Management of malignant ovarian germ cell tumors. Obstet Gynecol 
Surv 2011;66:507-14.

4. Young RH, Oliva E, Scully RE. Small cell carcinoma of the ovary, hypercalcemic type. Am J Surg Pathol 1994;18:1102-16.

5. Doubeni CA, Doubeni AR, Myers AE. Diagnosis and management of ovarian cancer. Am Fam Physician 2016;93:937-44.

6. Thigpen JT. Ovaries and Fallopian tubes. In: Abeloff MD, Armitage JO, Niederhuber JE, et al. editors. Clinical Oncology. 3rd. ed. Philadelphia: Elsevier, 2004:2312-38.

7. Sekiya S, Seki K, Nagai Y. Rise of serum CA 125 in patients with pure ovarian yolk sac tumors. Int J Gynaecol Obstet 1997;58:323-4.

8. Kim JH, Park JY, Kim JH, et al. The role of preoperative serum cancer antigen 125 in malignant ovarian germ cell tumors. Taiwan J Obstet Gynecol 2018;57:236-40.

9. Bagley CM Jr, Young RC, Canellos GP, et al. Treatment of ovarian carcinoma: possibilities for progress. $\mathrm{N} \mathrm{Engl} \mathrm{J}$ Med 1972;287:856-62.

10. McGowan L, Lesher LP, Norris HJ, et al. Mistaging of ovarian cancer. Obstet Gynecol 1985;65:568-72.

11. Mayer AR, Chambers SK, Graves E, et al. Ovarian cancer staging: does it require a gynecologic oncologist?. Gynecol Oncol 1992;47:223-7.

12. Ray-Coquard I, Morice P, Lorusso D, et al. Non-epithelial ovarian cancer: ESMO Clinical Practice Guidelines for diagnosis, treatment and follow-up. Ann Oncol 2018;29:iv1-18.

13. Reich H, McGlynn F, Wilkie W. Laparoscopic management of stage I ovarian cancer. A case report. J Reprod Med 1990;35:601-4; discussion 604-5.

14. Weber S, McCann CK, Boruta DM, et al. Laparoscopic surgical staging of early ovarian cancer. Rev Obstet Gynecol 2011;4:117-22.

15. Young RC, Decker DG, Wharton JT, et al. Staging laparotomy in early ovarian cancer. JAMA 1983;250:3072-6.

16. Lin KY, Bryant S, Miller DS, et al. Malignant ovarian germ cell tumor-role of surgical staging and gonadal dysgenesis. Gynecol Oncol 2014;134:84-9.

17. Chan JK, Tewari KS, Waller S, et al. The influence of conservative surgical practices for malignant ovarian germ cell tumors. J Surg Oncol 2008;98:111-6.

18. Mahdi H, Kumar S, Seward S, et al. Prognostic impact of laterality in malignant ovarian germ cell tumors. Int $\mathrm{J}$ Gynecol Cancer 2011;21:257-62.

19. Lee KH, Lee IH, Kim BG, et al. Clinicopathologic characteristics of malignant germ cell tumors in the ovaries of Korean women: a Korean gynecologic oncology group study. Int J Gynecol Cancer 2009;19:84-7.

20. Ertas IE, Taskin S, Goklu R, et al. Long-term oncological and reproductive outcomes of fertility-sparing cytoreductive surgery in females aged 25 years and younger with malignant ovarian germ cell tumors. J Obstet Gynaecol Res 2014;40:797-805.

21. Billmire D, Vinocur C, Rescorla F, et al. Outcome and staging evaluation in malignant germ cell tumors of the ovary in children and adolescents: an intergroup study. J Pediatr Surg 2004;39:424-9.

22. Gershenson DM, Frazier AL. Conundrums in the management of malignant ovarian germ cell tumors: toward lessening acute morbidity and late effects of treatment. Gynecol Oncol 2016;143:428-32.

23. Mangili G, Sigismondi C, Lorusso D, et al. Is surgical restaging indicated in apparent stage IA pure ovarian dysgerminoma? The MITO group retrospective experience. Gynecol Oncol 2011;121:280-4.

24. Nezhat FR, Ezzati M, Chuang L, et al. Laparoscopic management of early ovarian and fallopian tube cancers:surgical and survival outcome. Am J Obstet Gynecol 2009;200:83.e1-6.

25. Kumar S, Shah JP, Bryant CS, et al. The prevalence and prognostic impact of lymph node metastasis in malignant germ cell tumors of the ovary. Gynecol Oncol 2008;110:125-32.

26. Kuru O, Boyraz G, Uckan H, et al. Retroperitoneal nodal metastasis in primary adult type granulosa cell tumor of the ovary: can routine lymphadenectomy be omitted? Eur J Obstet Gynecol Reprod Biol 2017;219:70-3.

27. Mahdi H, Swensen RE, Hanna R, et al. Prognostic impact of lymphadenectomy in clinically early stage malignant germ cell tumour of the ovary. Br J Cancer 2011;105:493-7.

28. Liu Q, Ding X, Yang J, et al. The significance of comprehensive staging surgery in malignant ovarian germ cell tumors. Gynecol Oncol 2013;131:551-4.

29. Matei D, Brown J, Frazier L. Updates in the management of ovarian germ cell tumors. Am Soc Clin Oncol Educ Book 2013. doi: 10.1200/EdBook_AM.2013.33.e210.

30. Park JY, Kim DY, Suh DS, et al. Outcomes of pediatric and adolescent girls with malignant ovarian germ cell tumors. Gynecol Oncol 2015;137:418-22.

31. Hu T, Fang Y, Sun Q, et al. Clinical management of malignant ovarian germ cell tumors: a 26-year experience in a tertiary care institution. Surg Oncol 2019;31:8-13.

32. Lee YK, Park NH, Kim JW, et al. Characteristics of 
recurrence in adult-type granulosa cell tumor. Int J Gynecol Cancer 2008;18:642-7.

33. Nasioudis D, Ko EM, Haggerty AF, et al. Role of adjuvant chemotherapy in the management of stage IC ovarian granulosa cell tumors. Gynecol Oncol Rep 2019;28:145-8.

34. Bacalbasa N, Stoica C, Popa I, et al. Endometrial carcinoma associated with ovarian granulosa cell tumors--a case report. Anticancer Res 2015;35:5547-50.

35. Ukah CO, Ikpeze OC, Eleje GU, et al. Adult granulosa cell tumor associated with endometrial carcinoma: a case report. J Med Case Rep 2011;5:340.

36. Brown J, Sood AK, Deavers MT, et al. Patterns of metastasis in sex cord-stromal tumors of the ovary: can routine staging lymphadenectomy be omitted? Gynecol Oncol 2009;113:86-90.

37. Nasioudis D, Kanninen TT, Holcomb K, et al. Prevalence of lymph node metastasis and prognostic significance of lymphadenectomy in apparent early-stage malignant ovarian sex cord-stromal tumors. Gynecol Oncol 2017;145:243-7.

38. Wang J, Li J, Chen R, et al. Contribution of lymph node staging method and prognostic factors in malignant

Cite this article as: Medina-Franco H, Colonna-Márquez LE. Non-epithelial ovarian carcinoma: what is the optimal staging surgery? Chin Clin Oncol 2020;9(4):50. doi: 10.21037/cco-20-18 ovarian sex cord-stromal tumors: a world wide database analysis. Eur J Surg Oncol 2018;44:1054-61.

39. Dickersin GR, Kline IW, Scully RE. Small cell carcinoma of the ovary with hypercalcemia: a report of eleven cases. Cancer 1982;49:188-97.

40. Ramos P, Karnezis AN, Craig DW, et al. Small cell carcinoma of the ovary, hypercalcemic type, displays frequent inactivating germline and somatic mutations in SMARCA4. Nat Genet 2014;46:427-9.

41. Dykgraaf RHM, de Jong D, van Veen M, et al. Clinical management of ovarian small-cell carcinoma of the hypercalcemic type: a proposal for conservative surgery in an advanced stage of disease. Int J Gynecol Cancer 2009;19:348-53.

42. Lu B, Shi H. An in-depth look at small cell carcinoma of the ovary, hypercalcemic type (SCCOHT): clinical implications from recent molecular findings. J Cancer 2019;10:223-37.

43. Hurteau JA, Febbraro T. Germ cell tumors: treatment consensus across all age groups through MaGIC

[Malignant Germ Cell International Collaborative]. Cancer 2016;122:181-3. 